\title{
Space-dependent source determination in a time-fractional diffusion equation using a local discontinuous Galerkin method
}

\author{
S. Yeganeh ${ }^{1} \cdot$ R. Mokhtari ${ }^{1}$ J. S. Hesthaven ${ }^{2}$
}

the date of receipt and acceptance should be inserted later

\begin{abstract}
This paper is devoted to determining a space-dependent source term in an inverse problem of the time-fractional diffusion equation. We use a method based on a finite difference scheme in time and a local discontinuous Galerkin method (LDG) in space and investigate the numerical stability and convergence of the proposed method. Finally, various numerical examples are used illustrate the effectiveness and accuracy of the method.
\end{abstract}

Keywords Inverse source problem - fractional diffusion equation · local discontinuous Galerkin method

Mathematics Subject Classification (2000) 65M32 - 65M60 - 35R11

\section{Introduction}

In recent years, there has been an substantial research on problems involving the fractional order partial differential equations (PDEs), see e.g. [18,6, 24, 19,21, 17, 2,30, $25,29,22,27,28,31,8]$. In contrast to the classical diffusion equations, the fractional diffusion equations can be used to describe the anomalous diffusion phenomena such as super-diffusion or sub-diffusion. A time-fractional diffusion equation occurs when replacing the standard time derivative with a time fractional derivative and can be applied in modeling of some problems in porous flows, rheology and mechanical systems, models of a variety of biological processes, control and robotics, transport

Somayeh Yeganeh

E-mail: s.yeganeh@math.iut.ac.ir

Reza Mokhtari

E-mail: mokhtari@cc.iut.ac.ir

${ }^{1}$ Department of Mathematical Sciences, Isfahan University of Technology, Isfahan 84156-83111, Iran

Jan S Hesthaven

E-mail: Jan.Hesthaven@epfl.ch

2 EPFL-SB-MATH-MCSS, École Polytechnique Fédéral de Lausanne, CH-1015 Lausanne, Switzerland 
in fusion plasmas, and many other areas of applications. The direct problems corresponding to the time-fractional diffusion equations have been studied extensively in recent years, including uniqueness and existence results[2], some analytical or numerical solutions[13,7,31], and numerical methods such as finite element methods or finite difference methods $[12,14]$.

Here, we focus on an interesting inverse problem defined to the fractional inverse problem pioneered by Murio $[18,16,17]$. After that, many works have been published and we mention some of them in the following. In [2] an inverse problem has been considered to determine the order of the fractional derivative and the diffusion coefficient in a fractional diffusion equation. A uniqueness result has been also obtained. In [15], Liu et al. solved a backward problem for the time-fractional diffusion equation by a quasi-reversibility regularization method. Some Cauchy problems for the time-fractional diffusion equation on a bounded domain and on a strip have been investigated in $[35,36]$. A modified kernel method to deal with an inverse fractional diffusion equation has been presented in [19]. Wei et al. [25] have dealt with some space-fractional diffusion equations. Rundell et al. [21] have determined an unknown boundary condition in a fractional diffusion equation. We consider the following time-fractional diffusion equation [27],

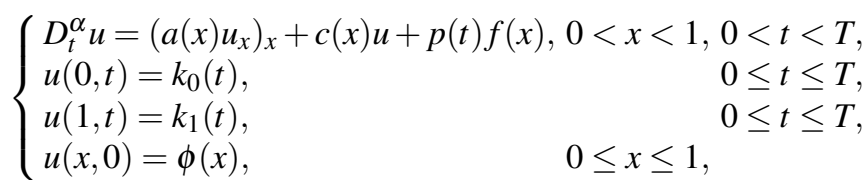

where the functions $a, c, p, k_{0}, k_{1}$ and $\phi$ are given and $u, f$ are unknown functions. To solve this inverse source problem we need an additional condition

$$
u(x, T)=g(x), \quad 0 \leq x \leq 1 .
$$

$D_{t}^{\alpha}$ is the Caputo fractional derivative of order $\alpha$, i.e.

$$
D_{t}^{\alpha} u(x, t)=\frac{1}{\Gamma(1-\alpha)} \int_{0}^{t} \frac{\partial u(x, s)}{\partial s} \frac{d s}{(t-s)^{\alpha}}, \quad 0<\alpha<1,
$$

where $\Gamma(\cdot)$ is the Gamma function. Obviously for $\alpha=1, D_{t}^{\alpha} u=u_{t}$. The existence of a unique weak solution for the direct problem (1.1) has been studied in [22] and the only uniqueness theorem for the inverse source problem mentioned above is the following.

Theorem 1.1 If $p \in C[0, T]$ satisfies $p(t) \geq p_{0}>0$ for all $t \in[0, T], a \in C^{1}[0,1]$ satisfies $a(x) \geq v>0$ for all $x \in[0,1]$, and $c \in C[0,1]$ satisfies $c(x) \leq 0$ for all $x \in[0,1]$ then the inverse source problem (1.1)-(1.3) has a unique solution $(u, f)$.

Theorem 1.1 has been proved in [27] where Wei et al. showed that the inverse source problem (1.1)-(1.2) is an ill-posed problem. For $\alpha=1$, we encounter a classical illposed problem which has been studied for example in [9,23], while for $0<\alpha<1$, to the best of our knowledge, only a few works have been carried out. Sakamoto et al. in [22] used additional data $u\left(x_{0}, t\right)$ for some given $x_{0}$ in the domain to determine $p$ when $f$ is given and the authors obtained Lipschitz stability for $p$. Wei et al. in 
[29] applied extra data to determine $p$ when $a=1, c=0$ and $f$ is given. This special case has been also considered in [33] where a local discontinuous Galerkin (LDG) method has been applied. In [35], a Fourier truncation method has been applied. In [26], for solving an inverse source problem with $p=1$ for one-dimensional case with special coefficients, a Tikhonov regularization method has been provided. In [27], Wei et al. focused on a multi-dimensional problem with variable coefficients in a general bounded domain. To the best of our knowledge, for the inverse source problem with or without fractional operators, there are no results to the DG methods and the development of the DG methods remains limited. Of course, the forward fractional diffusion equation has been solved successfully by some DG methods. For example, Wei et al. [28] have solved the time-fractional diffusion equation using a fully-discrete LDG method and some space-fractional diffusion equations have been solved by Hesthaven et al. [7,31] using a local discontinuous Galerkin method in a semi-discrete setting.

In this paper, we extend the application of the discontinuous Galerkin method to some inverse source problems. We present a local discontinuous Galerkin method for solving the inverse source problem of the time-fractional advection-diffusion equation (1.1)-(1.2). In the proposed method, the time-fractional derivative is discretized by a backward difference scheme and a LDG method is applied for the space variable.

The rest of the paper is organized as follows. At the end of this section, some preliminaries are provided. Section 2 is devoted to the construction of the proposed method for the inverse source problem (1.1)-(1.2) and the development of stability and convergence theorems of the proposed method. Some numerical examples are given in Section 3 to illustrate the accuracy and applicability of the method. Finally, a brief conclusion completes the paper.

First we decompose the domain of the problem into some cells (subintervals). For this purpose, we consider a mesh as $0=x_{\frac{1}{2}}<x_{\frac{3}{2}}<\cdots<x_{N+\frac{1}{2}}=1$ with cells $I_{j}=\left[x_{j-\frac{1}{2}}, x_{j+\frac{1}{2}}\right]$, for $j=1, \ldots, N . \Delta x_{j}=x_{j+\frac{1}{2}}-x_{j-\frac{1}{2}}$ denotes the cell length and we set $h=\max _{1 \leq j \leq N} \Delta x_{j}$. We denote by $u_{j+\frac{1}{2}}^{+}$and $u_{j+\frac{1}{2}}^{-}$, respectively the values of $u$ at $x_{j+\frac{1}{2}}$ from the right cell $I_{j+1}$ and from the left cell $I_{j}$. The jump of $u$ at cell interfaces is denoted by $[u]_{j+\frac{1}{2}}$, i.e. $u_{j+\frac{1}{2}}^{+}-u_{j+\frac{1}{2}}^{-}$. For some integer $k$, we set

$$
V_{h}^{k}=\left\{v \in L^{2}[0,1]|v|_{I_{j}} \in \mathbb{P}^{k}\left(I_{j}\right), j=1, \ldots, N\right\},
$$

as the space of polynomials of degree up to $k$ in each cell $I_{j}$.

We define projections $\mathbf{P}$ and $\mathbf{P}^{ \pm}$as follows

$$
\begin{aligned}
\int_{I_{j}}(\mathbf{P} \omega(x)-\omega(x)) v(x) & =0, \quad \forall v \in \mathbb{P}^{k}\left(I_{j}\right), \\
\int_{I_{j}}\left(\mathbf{P}^{+} \omega(x)-\omega(x)\right) v(x) & =0, \quad \forall v \in \mathbb{P}^{k-1}\left(I_{j}\right), \\
\mathbf{P}^{+} \omega\left(x_{j-\frac{1}{2}}^{+}\right) & =\omega\left(x_{j-\frac{1}{2}}\right),
\end{aligned}
$$


and

$$
\begin{aligned}
\int_{I_{j}}\left(\mathbf{P}^{-} \omega(x)-\omega(x)\right) v(x) & =0, \quad \forall v \in \mathbb{P}^{k-1}\left(I_{j}\right), \\
\mathbf{P}^{-} \omega\left(x_{j+\frac{1}{2}}^{+}\right) & =\omega\left(x_{j+\frac{1}{2}}\right) .
\end{aligned}
$$

These projections satisfy the inequality $[5,32]$

$$
\left\|\omega^{e}\right\|+h\left\|\omega^{e}\right\|_{\infty}+h^{\frac{1}{2}}\left\|\omega^{e}\right\|_{\tau_{h}} \leq C h^{k+1},
$$

where $\omega^{e}=\mathbf{P} \omega-\omega$ or $\omega^{e}=\mathbf{P}^{ \pm} \omega-\omega$, the positive constant $C$ depends solely on $\omega$ and is independent of $h, \tau_{h}$ denotes the set of boundary points of all elements $I_{j} .\|\cdot\|$ is the $L^{2}[0,1]$ norm.

\section{The LDG scheme}

In this section, we describe a numerical scheme for the solution of problem (1.1)(1.2). For some positive integer $M$, let $\Delta t=T / M$ be the time mesh size and $t_{n}=$ $n \Delta t, n=0, \ldots, M$ be the mesh points. The time fractional derivative (1.3) is approximated by a simple quadrature formula known as the L1 rule [13],

$$
D_{t}^{\alpha} u\left(x, t_{n}\right)=\frac{(\Delta t)^{1-\alpha}}{\Gamma(2-\alpha)} \sum_{i=0}^{n-1} b_{i} \frac{u\left(x, t_{n-i}\right)-u\left(x, t_{n-i-1}\right)}{\Delta t}+\gamma^{n}(x),
$$

where $b_{i}=(i+1)^{1-\alpha}-i^{1-\alpha}$ and $\gamma^{n}$ is the truncation error with the estimate [14]

$$
\left\|\gamma^{n}\right\| \leq C(\Delta t)^{2-\alpha} \text {. }
$$

Here $C$ is a constant depending on $u, \alpha$ and $T$. It is easy to check that $b_{i}>0$ for each $i, 1=b_{0}>b_{1}>\cdots$ and $b_{n} \rightarrow 0$ as $n \rightarrow \infty$.

We rewrite (1.1) as a first-order system

$$
q=u_{x}, \quad D_{t}^{\alpha} u(x, t)-(a(x) q)_{x}-c(x) u=f(x) p(t), \quad u(x, T)=g(x) .
$$

Let $u_{h}^{n}, q_{h}^{n}, f_{h} \in V_{h}^{k}$ be the approximation of $u\left(\cdot, t_{n}\right), q\left(\cdot, t_{n}\right), f(\cdot)$ respectively, and $p^{n}=$ $p\left(t_{n}\right)$. We define a local discontinuous Galerkin scheme as follows: find $u_{h}^{n}, q_{h}^{n} \in V_{h}^{k}$, such that for all test functions $v, w, y \in V_{h}^{k}$,

$$
\left\{\begin{array}{l}
\int_{\Omega} u_{h}^{n} v d x+\beta\left(\int_{\Omega}\left(a q_{h}^{n}\right) v_{x} d x-\sum_{j=1}^{N}\left(\left(\hat{a} \hat{q}_{h}^{n} v^{-}\right)_{j+\frac{1}{2}}-\left(\hat{a} \hat{q}_{h}^{n} v^{+}\right)_{j-\frac{1}{2}}\right)\right)- \\
\quad \beta \int_{\Omega} c u_{h}^{n} v d x=\beta p^{n} \int_{\Omega} f_{h} v d x+\sum_{i=1}^{n-1}\left(b_{i-1}-b_{i}\right) \int_{\Omega} u_{h}^{n-i} v d x+b_{n-1} \int_{\Omega} u_{h}^{0} v d x, \\
\int_{\Omega} q_{h}^{n} w d x+\int_{\Omega} u_{h}^{n} w_{x} d x-\sum_{j=1}^{N}\left(\left(\hat{u}_{h}^{n} w^{-}\right)_{j+\frac{1}{2}}-\left(\hat{u}_{h}^{n} w^{+}\right)_{j-\frac{1}{2}}\right)=0, \\
\int_{\Omega} u_{h}^{M} y d x=\int_{\Omega} g y d x,
\end{array}\right.
$$


where $\Omega=[0,1]$ and $\beta=(\Delta t)^{\alpha} \Gamma(2-\alpha)$. The "hat" terms in (2.2) are the "numerical fluxes", which are single valued functions defined on the edges. These are selected based on different guiding principles for different PDEs to ensure stability of the scheme. We take the following simple choice non-unique

$$
\hat{u}_{h}^{n}=\left(u_{h}^{n}\right)^{-}, \quad \hat{q}_{h}^{n}=\left(q_{h}^{n}\right)^{+},
$$

or

$$
\hat{u}_{h}^{n}=\left(u_{h}^{n}\right)^{+}, \quad \hat{q}_{h}^{n}=\left(q_{h}^{n}\right)^{-} .
$$

Following to $[3,4], \hat{u}_{h}^{n}$ and $\hat{q}_{h}^{n}$ must be taken from opposite sides. For convenience and without loss of generality, we consider the case $a(x)=1$ and $c(x)=0$. Using the first equation in (1.1) and equation (1.2), we have

$$
f(x)=\frac{1}{p^{M}}\left(\left.D_{t}^{\alpha} u\right|_{t=T}-g^{\prime \prime}(x)\right) .
$$

Lemma 2.1 If $u_{t}$ for all $t \in[0, T]$ is bounded and $g$ is sufficiently smooth with bounded derivatives, then $f$ will be bounded.

Proof At first, if $\left|u_{t}\right| \leq c$ then we have

$$
\begin{aligned}
\left|D_{t}^{\alpha} u(\cdot, T)\right| & =\left|\frac{1}{\Gamma(1-\alpha)} \int_{0}^{T} \frac{\partial u(\cdot, s)}{\partial s} \frac{d s}{(T-s)^{\alpha}}\right| \leq \frac{c}{\Gamma(1-\alpha)}\left|\int_{0}^{T} \frac{d s}{(T-s)^{\alpha}}\right| \\
= & \frac{c T^{(1-\alpha)}}{\Gamma(1-\alpha)}=C_{1},
\end{aligned}
$$

where $C_{1}$ is a constant that depends on $u, \alpha$ and $T$. From Theorem 1.1 and Eq. (2.4)

$$
|f(\cdot)|=\left|\frac{1}{p^{M}}\left(D_{t}^{\alpha} u(\cdot, T)-g^{\prime \prime}(\cdot)\right)\right| \leq \frac{1}{p_{0}}\left|\left(C_{1}-g^{\prime \prime}(\cdot)\right)\right| \leq C_{2},
$$

where $C_{2}$ is a constant depending on $u, g, \alpha$ and $T$.

Stability of the scheme (2.2) is given in the following result.

Theorem 2.1 If $u_{t}$ for all $t \in[0, T]$ is bounded and $g$ is sufficiently smooth with bounded derivatives, then for periodic or compactly supported boundary conditions, the fully-discrete LDG scheme (2.2) is unconditionally stable, and

$$
\left\|u_{h}^{n}\right\| \leq\left\|u_{h}^{0}\right\|+\kappa, \quad n=1, \ldots, M
$$

where $\kappa$ is a constant depending on $f$.

Proof We prove this theorem using induction. When $n=1,(2.2)$ is

$$
\begin{aligned}
\int_{\Omega} u_{h}^{1} v d x+ & \beta\left(\int_{\Omega} q_{h}^{1} v_{x} d x-\sum_{j=1}^{N}\left(\left(\hat{q}_{h}^{1} v^{-}\right)_{j+\frac{1}{2}}-\left(\hat{q}_{h}^{1} v^{+}\right)_{j-\frac{1}{2}}\right)\right)+\int_{\Omega} q_{h}^{1} w d x+\int_{\Omega} u_{h}^{1} w_{x} d x \\
& -\sum_{j=1}^{N}\left(\left(\hat{u}_{h}^{1} w^{-}\right)_{j+\frac{1}{2}}-\left(\hat{u}_{h}^{1} w^{+}\right)_{j-\frac{1}{2}}\right)=\beta p^{1} \int_{\Omega} f_{h} v d x+\int_{\Omega} u_{h}^{0} v d x .
\end{aligned}
$$


Taking test functions $v=u_{h}^{1}$ and $w=\beta q_{h}^{1}$, for periodic or compactly supported boundary conditions, we obtain

$$
\begin{gathered}
\beta\left(\int_{\Omega} q_{h}^{1} v_{x} d x-\sum_{j=1}^{N}\left(\left(\hat{q}_{h}^{1} v^{-}\right)_{j+\frac{1}{2}}-\left(\hat{q}_{h}^{1} v^{+}\right)_{j-\frac{1}{2}}\right)\right)-\sum_{j=1}^{N}\left(\left(\hat{u}_{h}^{1} w^{-}\right)_{j+\frac{1}{2}}-\left(\hat{u}_{h}^{1} w^{+}\right)_{j-\frac{1}{2}}\right) \\
+\int_{\Omega} u_{h}^{1} w_{x} d x=\beta \int_{\Omega} q_{h}^{1}\left(u_{h}^{1}\right)_{x} d x-\beta \sum_{j=1}^{N}\left(\left(\left(q_{h}^{1}\right)^{+}\left(u_{h}^{1}\right)^{-}\right)_{j+\frac{1}{2}}-\left(\left(q_{h}^{1}\right)^{+}\left(u_{h}^{1}\right)^{+}\right)_{j-\frac{1}{2}}\right) \\
+\beta \int_{\Omega} u_{h}^{1}\left(q_{h}^{1}\right)_{x} d x-\beta \sum_{j=1}^{N}\left(\left(\left(u_{h}^{1}\right)^{-}\left(q_{h}^{1}\right)^{-}\right)_{j+\frac{1}{2}}-\left(\left(u_{h}^{1}\right)^{-}\left(q_{h}^{1}\right)^{+}\right)_{j-\frac{1}{2}}\right)=0 .
\end{gathered}
$$

Then by using (2.3) and Lemma 2.1 we get

$$
\begin{aligned}
\left\|u_{h}^{1}\right\|^{2}+\beta \| q_{h}^{1} & \|^{2}=\int_{\Omega} u_{h}^{0} u_{h}^{1} d x+\beta p^{1} \int_{\Omega} f_{h} u_{h}^{1} d x=\int_{\Omega}\left(u_{h}^{0}+\beta p^{1} f_{h}\right) u_{h}^{1} d x \\
& \leq \frac{1}{2}\left(\left\|u_{h}^{0}+\beta p^{1} f_{h}\right\|^{2}+\left\|u_{h}^{1}\right\|^{2}\right) \\
& \leq \frac{1}{2}\left(\left(\left\|u_{h}^{0}\right\|+\beta p^{1}\left\|f_{h}\right\|\right)^{2}+\left\|u_{h}^{1}\right\|^{2}\right) \\
& \leq \frac{1}{2}\left(\left(\left\|u_{h}^{0}\right\|+\kappa\right)^{2}+\left\|u_{h}^{1}\right\|^{2}\right),
\end{aligned}
$$

Therefore

$$
\left\|u_{h}^{1}\right\| \leq\left\|u_{h}^{0}\right\|+\kappa .
$$

Now for the following inequality to hold

$$
\left\|u_{h}^{m}\right\| \leq\left\|u_{h}^{0}\right\|+\kappa, \quad m=1,2, \cdots, l,
$$

we must prove that $\left\|u_{h}^{l+1}\right\| \leq\left\|u_{h}^{0}\right\|+\kappa$. Let $n=l+1$ and take the test functions $v=u_{h}^{l+1}$ and $w=\beta u_{h}^{l+1}$ in scheme (2.2), and using

$$
\sum_{i=1}^{l}\left(b_{i-1}-b_{i}\right)+b_{l}=1
$$

to obtain

$$
\begin{aligned}
& \left\|u_{h}^{l+1}\right\|^{2}+\beta\left\|q_{h}^{l+1}\right\|^{2}=b_{l} \int_{\Omega} u_{h}^{0} u_{h}^{l+1} d x+\beta p^{l+1} \int_{\Omega} f_{h} u_{h}^{l+1} d x \\
& \quad+\sum_{i=1}^{l}\left(b_{i-1}-b_{i}\right) \int_{\Omega} u_{h}^{l+1-i} u_{h}^{l+1} d x=b_{l} \int_{\Omega}\left(u_{h}^{0}+\frac{\beta p^{l+1}}{b_{l}} f_{h}\right) u_{h}^{l+1} d x \\
& \quad+\sum_{i=1}^{l}\left(b_{i-1}-b_{i}\right) \int_{\Omega} u_{h}^{l+1-i} u_{h}^{l+1} d x \leq b_{l}\left(\left\|u_{h}^{0}\right\|+\kappa\right)\left\|u_{h}^{l+1}\right\| \\
& \quad+\sum_{i=1}^{l}\left(b_{i-1}-b_{i}\right)\left(\left\|u_{h}^{0}\right\|+\kappa\right)\left\|u_{h}^{l+1}\right\|=\left(\left\|u_{h}^{0}\right\|+\kappa\right)\left\|u_{h}^{l+1}\right\| \\
& \quad \leq \frac{1}{2}\left(\left(\left\|u_{h}^{0}\right\|+\kappa\right)^{2}+\left\|u_{h}^{l+1}\right\|^{2}\right)
\end{aligned}
$$

and then

$$
\left\|u_{h}^{l+1}\right\| \leq\left\|u_{h}^{0}\right\|+\kappa
$$


Convergence of the scheme is given as.

Theorem 2.2 Let $u\left(\cdot, t_{n}\right)$ be the exact solution of $(1.1), u_{h}^{n}$ be the numerical solution of the fully discrete LDG scheme (2.2), g be sufficiently smooth with bounded derivatives and $u_{t}$ for all $t \in[0, T]$ be bounded. Then there holds the following error estimate

$$
\left\|u\left(\cdot, t_{n}\right)-u_{h}^{n}\right\| \leq C\left(h^{k+1}+(\Delta t)^{2}+(\Delta t)^{\frac{\alpha}{2}} h^{k+\frac{1}{2}}\right)+\tilde{C}(\Delta t)^{\frac{\alpha}{2}} h^{k+1}
$$

where $C$ is a constant depending on $u, \alpha, T$ and $\tilde{C}$ on $f, p$.

Proof We denote

$$
\begin{aligned}
& e_{u}^{n}=u\left(x, t_{n}\right)-u_{h}^{n}=\mathbf{P}^{-} e_{u}^{n}-\left(\mathbf{P}^{-} u\left(x, t_{n}\right)-u\left(x, t_{n}\right)\right) \\
& e_{q}^{n}=q\left(x, t_{n}\right)-q_{h}^{n}=\mathbf{P} e_{q}^{n}-\left(\mathbf{P} q\left(x, t_{n}\right)-q\left(x, t_{n}\right)\right)
\end{aligned}
$$

It is easy to verify that the exact solution of (2.2) satisfies

$$
\begin{aligned}
\int_{\Omega} u\left(x, t_{n}\right) v d x & +\beta\left(\int_{\Omega} q\left(x, t_{n}\right) v_{x} d x-\sum_{j=1}^{N}\left(\left(q\left(x, t_{n}\right) v^{-}\right)_{j+\frac{1}{2}}-\left(q\left(x, t_{n}\right) v^{+}\right)_{j-\frac{1}{2}}\right)\right) \\
& +\int_{\Omega} u\left(x, t_{n}\right) w_{x} d x-\sum_{j=1}^{N}\left(\left(u\left(x, t_{n}\right) w^{-}\right)_{j+\frac{1}{2}}-\left(u\left(x, t_{n}\right) w^{+}\right)_{j-\frac{1}{2}}\right) \\
& -\sum_{i=1}^{n-1}\left(b_{i-1}-b_{i}\right) \int_{\Omega} u\left(x, t_{n-i}\right) v d x-b_{n-1} \int_{\Omega} u\left(x, t_{0}\right) v d x+\int_{\Omega} q\left(x, t_{n}\right) w d x \\
+ & \beta \int_{\Omega} \gamma^{n}(x) v d x-\beta p^{n} \int_{\Omega} f(x) v d x=0 .
\end{aligned}
$$

Subtracting equation (2.2) from (2.10), we obtain the error equation

$$
\begin{aligned}
\int_{\Omega} e_{u}^{n} v d x & +\beta\left(\int_{\Omega} e_{q}^{n} v_{x} d x-\sum_{j=1}^{N}\left(\left(\left(e_{q}^{n}\right)^{+} v^{-}\right)_{j+\frac{1}{2}}-\left(\left(e_{q}^{n}\right)^{+} v^{+}\right)_{j-\frac{1}{2}}\right)\right)+\int_{\Omega} e_{q}^{n} w d x \\
& +\int_{\Omega} e_{u}^{n} w_{x} d x-\sum_{j=1}^{N}\left(\left(\left(e_{u}^{n}\right)^{-} w^{-}\right)_{j+\frac{1}{2}}-\left(\left(e_{u}^{n}\right)^{-} w^{+}\right)_{j-\frac{1}{2}}\right)-b_{n-1} \int_{\Omega} e_{u}^{0} v d x \\
& -\sum_{i=1}^{n-1}\left(b_{i-1}-b_{i}\right) \int_{\Omega} e_{u}^{n-i} v d x+\beta \int_{\Omega} \gamma^{n}(x) v d x-\beta p^{n} \int_{\Omega}\left(f(x)-f_{h}\right) v d x=0
\end{aligned}
$$


Using equation (2.9), the error equation (2.10) can be written

$$
\begin{aligned}
& \int_{\Omega} \mathbf{P}^{-} e_{u}^{n} v d x+\beta\left(\int_{\Omega} \mathbf{P} e_{q}^{n} v_{x} d x-\sum_{j=1}^{N}\left(\left(\left(\mathbf{P} e_{q}^{n}\right)^{+} v^{-}\right)_{j+\frac{1}{2}}-\left(\left(\mathbf{P} e_{q}^{n}\right)^{+} v^{+}\right)_{j-\frac{1}{2}}\right)\right) \\
& +\int_{\Omega} \mathbf{P} e_{q}^{n} w d x+\int_{\Omega} \mathbf{P}^{-} e_{u}^{n} w_{x} d x-\sum_{j=1}^{N}\left(\left(\left(\mathbf{P}^{-} e_{u}^{n}\right)^{-} w^{-}\right)_{j+\frac{1}{2}}-\left(\left(\mathbf{P}^{-} e_{u}^{n}\right)^{-} w^{+}\right)_{j-\frac{1}{2}}\right) \\
& -\beta p^{n} \int_{\Omega}\left(f(x)-f_{h}\right) v d x=b_{n-1} \int_{\Omega} \mathbf{P}^{-} e_{u}^{0} v d x+\sum_{i=1}^{n-1}\left(b_{i-1}-b_{i}\right) \int_{\Omega} \mathbf{P}^{-} e_{u}^{n-i} v d x \\
& -\beta \int_{\Omega} \gamma^{n}(x) v d x+\int_{\Omega}\left(\mathbf{P}^{-} u\left(x, t_{n}\right)-u\left(x, t_{n}\right)\right) v d x+\beta\left(\int_{\Omega}\left(\mathbf{P} q\left(x, t_{n}\right)-q\left(x, t_{n}\right)\right) v_{x} d x\right. \\
& \left.-\sum_{j=1}^{N}\left(\left(\left(\mathbf{P} q\left(x, t_{n}\right)-q\left(x, t_{n}\right)\right)^{+} v^{-}\right)_{j+\frac{1}{2}}-\left(\left(\mathbf{P}_{q} q\left(x, t_{n}\right)-q\left(x, t_{n}\right)\right)^{+} v^{+}\right)_{j-\frac{1}{2}}\right)\right) \\
& +\int_{\Omega}\left(\mathbf{P} q\left(x, t_{n}\right)-q\left(x, t_{n}\right)\right) w d x+\int_{\Omega}\left(\mathbf{P}^{-} u\left(x, t_{n}\right)-u\left(x, t_{n}\right)\right) w_{x} d x \\
& -\sum_{j=1}^{N}\left(\left(\left(\mathbf{P}^{-} u\left(x, t_{n}\right)-u\left(x, t_{n}\right)\right)^{-} w^{-}\right)_{j+\frac{1}{2}}-\left(\left(\mathbf{P}^{-} u\left(x, t_{n}\right)-u\left(x, t_{n}\right)\right)^{-} w^{+}\right)_{j-\frac{1}{2}}\right) \\
& -b_{n-1} \int_{\Omega}\left(\mathbf{P}^{-} u\left(x, t_{0}\right)-u\left(x, t_{0}\right)\right) v d x-\sum_{i=1}^{n-1}\left(b_{i-1}-b_{i}\right) \int_{\Omega}\left(\mathbf{P}^{-} u\left(x, t_{n-i}\right)-u\left(x, t_{n-i}\right)\right) v d x .
\end{aligned}
$$

Taking test functions $v=u_{h}^{n}$ and $w=\beta q_{h}^{n}$, for periodic or compactly supported boundary conditions, we obtain

$$
\begin{array}{rl}
\int_{\Omega}\left(\mathbf{P}^{-} e_{u}^{n}\right)^{2} & d x+\int_{\Omega}\left(\mathbf{P} e_{q}^{n}\right)^{2} d x-\beta p^{n} \int_{\Omega}\left(f(x)-f_{h}\right) \mathbf{P}^{-} e_{u}^{n} d x \\
& =b_{n-1} \int_{\Omega} \mathbf{P}^{-} e_{u}^{0} \mathbf{P}^{-} e_{u}^{n} d x+\sum_{i=1}^{n-1}\left(b_{i-1}-b_{i}\right) \int_{\Omega} \mathbf{P}^{-} e_{u}^{n-i} \mathbf{P}^{-} e_{u}^{n} d x \\
& -\beta \int_{\Omega} \gamma^{n}(x) \mathbf{P}^{-} e_{u}^{n} d x+\beta \sum_{j=1}^{N}\left(\left(\left(\mathbf{P} q\left(x, t_{n}\right)-q\left(x, t_{n}\right)\right)^{+}\left[\mathbf{P}^{-} e_{u}^{n}\right]\right)_{j-\frac{1}{2}}\right. \\
& +\int_{\Omega}\left(\mathbf{P}^{-} u\left(x, t_{n}\right)-u\left(x, t_{n}\right)\right) \mathbf{P}^{-} e_{u}^{n} d x-b_{n-1} \int_{\Omega}\left(\mathbf{P}^{-} u\left(x, t_{0}\right)-u\left(x, t_{0}\right)\right) \mathbf{P}^{-} e_{u}^{n} d x \\
& -\sum_{i=1}^{n-1}\left(b_{i-1}-b_{i}\right) \int_{\Omega}\left(\mathbf{P}^{-} u\left(x, t_{n-i}\right)-u\left(x, t_{n-i}\right)\right) \mathbf{P}^{-} e_{u}^{n} d x .
\end{array}
$$

For $n=1$, we have

$$
\begin{array}{rl}
\int_{\Omega}\left(\mathbf{P}^{-} e_{u}^{1}\right)^{2} & d x+\beta \int_{\Omega}\left(\mathbf{P} e_{q}^{1}\right)^{2} d x-\beta p^{1} \int_{\Omega}\left(f(x)-f_{h}\right) \mathbf{P}^{-} e_{u}^{1} d x=\int_{\Omega} \mathbf{P}^{-} e_{u}^{0} \mathbf{P}^{-} e_{u}^{1} d x \\
& -\beta \int_{\Omega} \gamma^{1}(x) \mathbf{P}^{-} e_{u}^{1} d x+\beta \sum_{j=1}^{N}\left(\left(\left(\mathbf{P} q\left(x, t_{1}\right)-q\left(x, t_{1}\right)\right)^{+}\left[\mathbf{P}^{-} e_{u}^{1}\right]\right)_{j-\frac{1}{2}}\right. \\
& +\int_{\Omega}\left(\mathbf{P}^{-} u\left(x, t_{1}\right)-u\left(x, t_{1}\right)\right) \mathbf{P}^{-} e_{u}^{1} d x-\int_{\Omega}\left(\mathbf{P}^{-} u\left(x, t_{0}\right)-u\left(x, t_{0}\right)\right) \mathbf{P}^{-} e_{u}^{1} d x
\end{array}
$$

Recalling that

$\left\|P^{-} e_{u}^{0}\right\| \leq C h^{k+1}, \quad\left\|P^{-} e_{u}^{1}\right\| \leq C h^{k+1}, \quad\left\|f(x)-f_{h}\right\| \leq c h^{k+1}\|f\|, \quad a b \leq \varepsilon a^{2}+\frac{1}{4 \varepsilon} b^{2}$, 
we can write

$$
\begin{aligned}
\left\|\mathbf{P}^{-} e_{u}^{1}\right\|^{2} & +\beta\left\|\mathbf{P} e_{q}^{1}\right\|^{2} \leq\left(\left\|\mathbf{P}^{-} e_{u}^{0}\right\|+\beta\left\|\gamma^{1}(x)\right\|+\left\|\mathbf{P}^{-} u\left(x, t_{1}\right)-u\left(x, t_{1}\right)\right\|\right. \\
& \left.+\left\|\mathbf{P}^{-} u\left(x, t_{0}\right)-u\left(x, t_{0}\right)\right\|\right)\left\|\mathbf{P}^{-} e_{u}^{1}\right\|+\beta p^{1}\left\|f(x)-f_{h}\right\|\left\|\mathbf{P}^{-} e_{u}^{1}\right\| \| \\
& +\frac{\beta}{4} \sum_{j=1}^{N}\left(\left(\mathbf{P} q\left(x, t_{1}\right)-q\left(x, t_{1}\right)\right)^{+}\right)_{j-\frac{1}{2}}^{2}+\varepsilon \beta \sum_{j=1}^{N}\left[\mathbf{P}^{-} e_{u}^{1}\right]_{j-\frac{1}{2}} \\
& \leq C\left(h^{k+1}+(\Delta t)^{2}+(\Delta t)^{\frac{\alpha}{2}} h^{k+\frac{1}{2}}\right)^{2}+\varepsilon\left\|\mathbf{P}^{-} e_{u}^{1}\right\|^{2} \\
& +c(\Delta t)^{\alpha} p^{1} h^{2 k+2}\|f\|+\varepsilon \beta \sum_{j=1}^{N}\left[\mathbf{P}^{-} e_{u}^{1}\right]_{j-\frac{1}{2}} .
\end{aligned}
$$

If we choose $\varepsilon$ very small and use Lemma 2.1, we obtain

$$
\left\|\mathbf{P}^{-} e_{u}^{1}\right\|^{2}+\beta\left\|\mathbf{P} e_{q}^{1}\right\|^{2} \leq C\left(h^{k+1}+(\Delta t)^{2}+(\Delta t)^{\frac{\alpha}{2}} h^{k+\frac{1}{2}}\right)^{2}+\tilde{C}(\Delta t)^{\alpha} h^{2 k+2},
$$

where $\tilde{C}$ is a constant independent of $h$, and

$$
\left\|\mathbf{P}^{-} e_{u}^{m}\right\|_{\Omega} \leq C\left(h^{k+1}+(\Delta t)^{2}+(\Delta t)^{\alpha} h^{k+\frac{1}{2}}\right)+\tilde{C}(\Delta t)^{\frac{\alpha}{2}} h^{k+1}, \quad m=1,2, \ldots, K .
$$

Using Eq. (2.12) and choosing a small enough $\varepsilon$, we obtain

$$
\left\|\mathbf{P}^{-} e_{u}^{K+1}\right\| \leq C\left(h^{k+1}+(\Delta t)^{2}+(\Delta t)^{\frac{\alpha}{2}} h^{k+\frac{1}{2}}\right)+\tilde{C}(\Delta t)^{\frac{\alpha}{2}} h^{k+1} .
$$

\section{Implementation details}

Here, we aim to describe our method somewhat more. We set

$$
\mathbb{P}^{k}\left(I_{j}\right)=\operatorname{Span}\left\{\phi_{1}, \ldots, \phi_{k}\right\}
$$

with

$$
\phi_{1}(x)=1, \quad \phi_{2}(x)=\left(2 \frac{x-\bar{x}_{j}}{\Delta x_{j}}\right), \quad \ldots, \quad \phi_{k}(x)=\left(2 \frac{x-\bar{x}_{j}}{\Delta x_{j}}\right)^{k},
$$

and $\bar{x}_{j}=\frac{1}{2}\left(x_{j+\frac{1}{2}}+x_{j-\frac{1}{2}}\right)$ is the midpoint of the interval $I_{j}$. The global basis functions $\Phi_{1}, \ldots, \Phi_{N_{p}}$ for the space $V_{h}^{k}$ are obtained from the local basis functions by extending them by zero, i.e.

$$
\Phi_{(j-1) k+i}(x)= \begin{cases}\phi_{i}(x), & x \in I_{j}, \\ 0, & x \notin I_{j},\end{cases}
$$

for $i=1, \ldots, k$ and $j=1, \ldots, N$.

We set

$$
u_{h}^{n}(x)=u_{h}(x, n \Delta t)=\sum_{i=1}^{N_{p}} \delta_{i}^{n} \Phi_{i}(x), \quad q_{h}^{n}(x)=q_{h}(x, n \Delta t)=\sum_{i=1}^{N_{p}} \gamma_{i}^{n} \Phi_{i}(x),
$$

where $N_{p}$ is the total number of basis functions and

$$
F=\left(\int_{\Omega} f_{h}(x) \Phi_{1}(x) d x \cdots \int_{\Omega} f_{h}(x) \Phi_{N_{p}}(x) d x\right)^{T},
$$




$$
G=\left(\int_{\Omega} g(x) \Phi_{1}(x) d x \cdots \int_{\Omega} g(x) \Phi_{N_{p}}(x) d x\right)^{T} .
$$

Setting $\delta^{n}=\left(\delta_{1}^{n} \cdots \delta_{N_{p}}^{n}\right)^{T}$ and $\gamma^{n}=\left(\gamma_{1}^{n} \cdots \gamma_{N_{p}}^{n}\right)^{T}$, scheme (2.2) leads to the following iteration scheme

$$
\left\{\begin{array}{l}
K_{11} \delta^{n}+K_{12} \gamma^{n}=\beta p^{n} F+\sum_{i=1}^{n-1}\left(b_{i-1}-b_{i}\right) K_{22} \delta^{n-i} \\
K_{21} \delta^{n}+K_{22} \gamma^{n}=0 \\
K_{22} \delta^{M}=G
\end{array}\right.
$$

where $n=1, \ldots, M$ and

$$
\begin{gathered}
\left(K_{11}\right)_{l r}=\int_{\Omega} \Phi_{l}(x) \Phi_{r}(x) d x-\beta \int_{\Omega} c(x) \Phi_{l}(x) \Phi_{r}(x) d x, \\
\left(K_{12}\right)_{l r}=\beta \int_{\Omega} a(x) \Phi_{l}(x)\left(\Phi_{r}(x)\right)_{x} d x \\
-\beta \sum_{j=1}^{N}\left(a\left(x_{j+\frac{1}{2}}^{-}\right) \Phi_{l}\left(x_{j+\frac{1}{2}}^{-}\right) \Phi_{r}\left(x_{j+\frac{1}{2}}^{-}\right)-a\left(x_{j-\frac{1}{2}}^{-}\right) \Phi_{l}\left(x_{j-\frac{1}{2}}^{-}\right) \Phi_{r}\left(x_{j-\frac{1}{2}}^{+}\right)\right), \\
\left(K_{21}\right)_{l r}=\int_{\Omega} \Phi_{l}(x)\left(\Phi_{r}(x)\right)_{x} d x-\sum_{j=1}^{N}\left(\Phi_{l}\left(x_{j+\frac{1}{2}}^{+}\right) \Phi_{r}\left(x_{j+\frac{1}{2}}^{-}\right)-\Phi_{l}\left(x_{j-\frac{1}{2}}^{+}\right) \Phi_{r}\left(x_{j-\frac{1}{2}}^{+}\right)\right), \\
\left(K_{22}\right)_{l r}=\int_{\Omega} \Phi_{l}(x) \Phi_{r}(x) d x .
\end{gathered}
$$

For solving the direct problem, we have

$$
M\left(\begin{array}{l}
\delta^{n} \\
\gamma^{n}
\end{array}\right)=\left(\begin{array}{c}
0 \\
\beta p^{n} F+\sum_{i=1}^{n-1}\left(b_{i-1}-b_{i}\right) K_{22} \delta^{n-i}
\end{array}\right), \quad M=\left(\begin{array}{ll}
K_{11} & K_{12} \\
K_{21} & K_{22}
\end{array}\right),
$$

where matrix $K_{22}$ is nonsingular and block diagonal which every block is a $k \times k$ ( $k$ is degree of basis polynomials) matrix and if $1-\beta c(x) \geq 0$ then $K_{11}$ is positive definite and clearly nonsingular.

For solving the inverse problem, using the first and second equations in (3.1), we have

$$
K \delta^{n}=\beta p^{n} F+\sum_{i=1}^{n-1}\left(b_{i-1}-b_{i}\right) K_{22} \delta^{n-i},
$$

where $K=K_{11}-K_{12} K_{22}^{-1} K_{21}$. Provided $M$ is invertible, the positive definiteness of $K_{11}$ and $K_{22}$ guarantees that $K$ is invertible [1]. Furthermore,

$$
\left\{\begin{array}{lrl}
\delta^{1}=K^{-1} \beta p^{1} F=A_{1} F, & n & =1, \\
\delta^{2}=K^{-1}\left(\beta p^{2} F+\left(b_{0}-b_{1}\right) K_{22} \alpha^{1}\right)=A_{2} F & n & =2, \\
\vdots & & n=M-1 .
\end{array}\right.
$$


For $n=M$, using numerical fluxes $\hat{u}_{h}^{M}=g(x)$ and $\hat{q}_{h}^{M}=g^{\prime}(x)$, by defining the following vectors

$$
\begin{aligned}
\left(G_{1}\right)_{l} & =\sum_{j=1}^{N}\left(g\left(x_{j+\frac{1}{2}}^{-}\right) \Phi_{l}\left(x_{j+\frac{1}{2}}^{-}\right)-g\left(x_{j-\frac{1}{2}}^{-}\right) \Phi_{l}\left(x_{j-\frac{1}{2}}^{+}\right)\right), \\
\left(G_{2}\right)_{l} & =\sum_{j=1}^{N}\left(g^{\prime}\left(x_{j+\frac{1}{2}}^{+}\right) \Phi_{l}\left(x_{j+\frac{1}{2}}^{-}\right)-g^{\prime}\left(x_{j-\frac{1}{2}}^{+}\right) \Phi_{l}\left(x_{j-\frac{1}{2}}^{+}\right)\right),
\end{aligned}
$$

we obtain

$$
\left\{\begin{array}{l}
K_{11} \delta^{M}+\beta \bar{K}_{12} \gamma^{M}-\beta G_{1}=\beta p^{M} F+\sum_{i=1}^{M-1}\left(b_{i-1}-b_{i}\right) K_{1} \delta^{M-i} \\
\bar{K}_{21} \delta^{M}+K_{22} \gamma^{M}-G_{2}=0 \\
K_{22} \delta^{M}=G
\end{array}\right.
$$

where

$$
\left(\bar{K}_{12}\right)_{l r}=\int_{\Omega} a(x) \Phi_{l}(x)\left(\Phi_{r}(x)\right)_{x} d x, \quad\left(\bar{K}_{21}\right)_{l r}=\int_{\Omega} \Phi_{l}(x)\left(\Phi_{r}(x)\right)_{x} d x
$$

Finally, we have

$$
A_{M} F=K_{M} G-\beta G_{1}+\beta \bar{K}_{12} K_{22}^{-1} G_{2}, \quad K_{M}=\left(K_{11}-\beta \bar{K}_{12} K_{22}^{-1} \bar{K}_{21}\right) K_{22}^{-1} .
$$

Solving the above linear system, we obtain $F$ and by applying a suitable integration rule we recover $f_{h}$ as an approximation of the source term. Through (3.3), we find the coefficients of $u_{h}$ at each time step and obtain

$$
u_{h}^{n}(x)=u_{h}(x, n \Delta t)=\sum_{i=1}^{N_{p}} \delta_{i}^{n} \Phi_{i}(x) .
$$

\section{Numerical examples}

In this section, we present some numerical results to investigate the effectiveness and stability of the proposed method. The maximum time is $T=1$ unless otherwise specified. The space and time step is $h=1 / N$ and $\Delta t=1 / M$, respectively. For noisy data, we use

$$
g^{\delta}\left(x_{i}\right)=g\left(x_{i}\right)+\varepsilon g\left(x_{i}\right)(2 \operatorname{rand}(\mathrm{i})-1),
$$

where $g\left(x_{i}\right)$ is the exact data, $\operatorname{rand}(\mathrm{i})$ is an uniformly distributed random number in $[0,1]$ and the magnitude $\varepsilon$ indicates a relative noise level. The corresponding noise level is calculated $\delta=\left\|g^{\delta}-g\right\|$. To measure the accuracy of the numerical solutions, we compute the approximate $L^{2}$ error as

$$
e(f, \varepsilon)=\left\|f-f^{\delta}\right\|
$$

and the approximate relative error in the $L^{2}$-norm as

$$
e_{r}(f, \varepsilon)=\left\|f-f^{\delta}\right\| /\|f\| \text {. }
$$


Table 4.1 Accuracy test for Example 4.1 for different $\alpha$

\begin{tabular}{cccccc}
\hline & $n$ & $L^{2}$ error & CO & $L^{\infty}$ error & CO \\
& & & & & \\
\hline$\alpha=0.3$ & 5 & 0.005563 & - & 0.007862 & - \\
& 10 & 0.000722 & 2.945792363366441 & 0.000976 & 3.009943310861208 \\
& 15 & 0.000215 & 2.987648225558304 & 0.000305 & 2.868682869489701 \\
& 20 & 0.000091 & 2.988641295174632 & 0.000127 & 3.045461549557176 \\
$\alpha=0.5$ & 5 & 0.005390 & - & 0.007618 & - \\
& 10 & 0.000717 & 2.910240248937963 & 0.000969 & 2.974843717148259 \\
& 15 & 0.000215 & 2.970509147116684 & 0.000304 & 2.859030006042589 \\
& 20 & 0.000091 & 2.988641295174632 & 0.000127 & 3.034045908765933 \\
$\alpha=0.7$ & 5 & 0.004832 & - & 0.006828 & - \\
& 10 & 0.000697 & 2.793389893456656 & 0.000942 & 2.857664093414955 \\
& 15 & 0.000212 & 2.935392250252534 & 0.000300 & 2.822000653173150 \\
& 20 & 0.000090 & 2.978206452142942 & 0.000216 & 3.015483586833959 \\
& 10 & 0.000574 & - & 0.000777 & - \\
& 20 & 0.000085 & 2.755515990537986 & 0.000120 & 2.694880192799192 \\
& 30 & 0.000026 & 2.921471403534026 & 0.000037 & 2.901788111010409 \\
& 40 & 0.000011 & 2.990110777122867 & 0.000016 & 2.914082143735104 \\
\hline
\end{tabular}

To verify the convergence rate, we use the following definition

$$
\mathrm{CO}=\log _{2} \frac{\mathrm{e}(\mathrm{f}, 2 \varepsilon)}{\mathrm{e}(\mathrm{f}, \varepsilon)}
$$

Example 4.1 Consider the inverse source problem of the time-fractional diffusion equation (1.1) with the exact solution $u(x, t)=t^{2} \sin (2 \pi x)$ and $f(x)=\sin (2 \pi x), p(t)=$ $(2 \pi t)^{2}+\frac{2 t^{2-\alpha}}{\Gamma(3-\alpha)}$. We take piecewise $P^{2}$ polynomials as the basis functions. Setting $\Delta t$ very small, we show in Table 4.1 that the order of convergence of the proposed method for several $\alpha$ is about three as we expected. In Fig. 1, we show the errors in $L^{2}$-norm and $L^{\infty}$-norm for piecewise $P^{k}, k=1,2,3$ polynomials for $\alpha=0.5$.

In order to investigate the impact of the "inverse crime", we consider the following two cases for solving the proposed problem. We consider $k=1$ for both cases.

Case 1: We use $g(x)=\sin (2 \pi x)$ and solve the inverse problem using the proposed LDG method. Errors of the method are shown in Table 4.2.

Case 2: We first solve a direct problem using an LDG method to obtain the input data $g$ and solve the inverse problem using the proposed method. We use a finer grid to solve the forward problem, i.e. we take $N=100$ (the grid points for the space interval) and $M=200$ (the grid points for the time interval) and choose $N=50$ and $M=200$ for solving the inverse problem. Errors of the method are shown in Table 4.3.

It can be seen that for both cases the numerical solutions obtained by the LDG method are not sensitive with respect to the perturbation in the initial data and we do not observe the impact of the "inverse crime".

Example 4.2 Consider the inverse source problem of the time-fractional diffusion equation (1.1) with $a(x)=x^{2}+1$ and $c(x)=-(x+1)$. Take a source function $p(t)=$ $e^{-t}$ and $f(x)=(x(1-x))^{\alpha} \sin (5 \pi x)$. Since the exact solution of this problem is not 

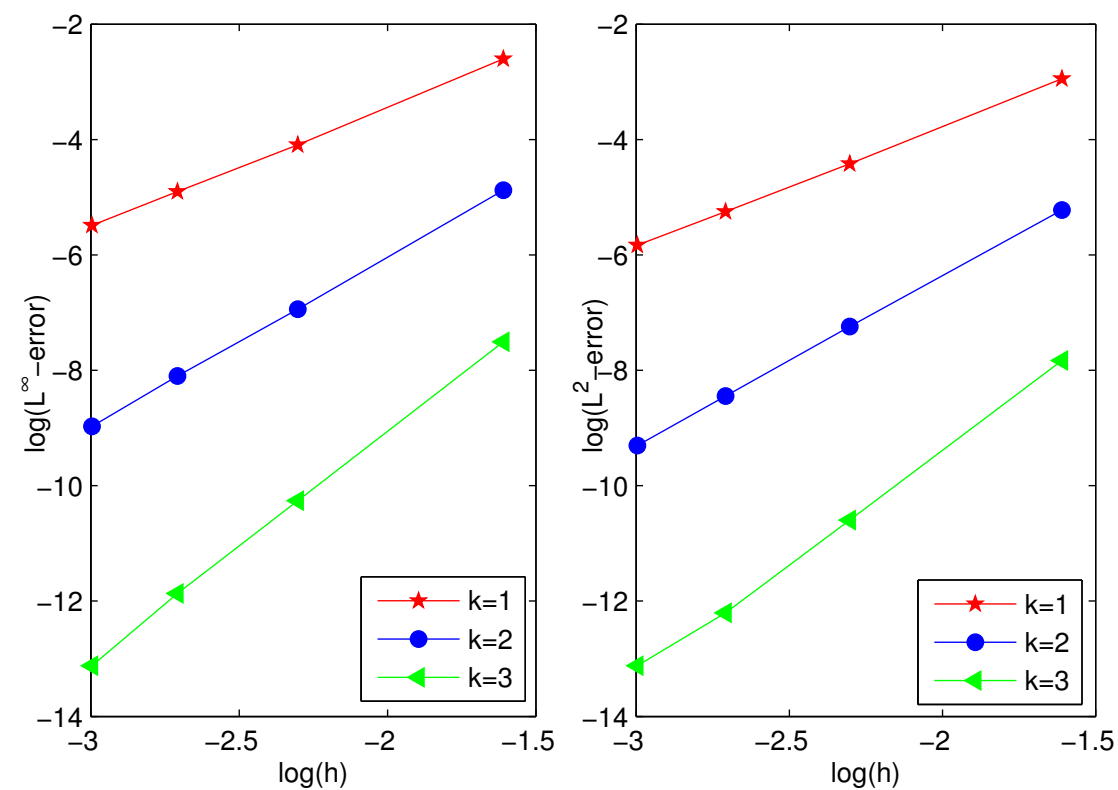

Fig. 4.1 $\log$ (error) as a function of $\log (\mathrm{h})$ for $\alpha=0.5$ when using piecewise $P^{k}, k=1,2,3$ polynomials for Example 4.1

Table 4.2 Errors of the LDG method (case 1) for example 1 for different $\alpha$ with $\varepsilon=0.01$ (left) and for different $\varepsilon$ with $\alpha=0.6$ (right).

\begin{tabular}{crc} 
& & \\
$\alpha$ & $e(f, 0.01)$ & $e_{r}(f, 0.01)$ \\
\hline 0.05 & $1.00 \times 10^{-5}$ & $9.88 \times 10^{-6}$ \\
0.1 & $1.60 \times 10^{-5}$ & $1.59 \times 10^{-5}$ \\
0.3 & $8.00 \times 10^{-5}$ & $7.96 \times 10^{-5}$ \\
0.7 & $8.22 \times 10^{-4}$ & $8.22 \times 10^{-4}$ \\
0.9 & $2.27 \times 10^{-3}$ & $2.26 \times 10^{-3}$ \\
0.95 & $2.90 \times 10^{-3}$ & $2.90 \times 10^{-3}$ \\
\hline
\end{tabular}

\begin{tabular}{crr}
\hline & & \\
$\varepsilon$ & $e(f, \varepsilon)$ & $e_{r}(f, \varepsilon)$ \\
\hline 0.0005 & 0.000471 & 0.000470948 \\
0.001 & 0.000471 & 0.000471018 \\
0.002 & 0.000471 & 0.000471459 \\
0.004 & 0.000472 & 0.000472316 \\
0.008 & 0.000479 & 0.000479103 \\
0.016 & 0.000491 & 0.000491375 \\
0.032 & 0.000556 & 0.000555792 \\
0.064 & 0.000770 & 0.000770034 \\
\hline
\end{tabular}

Table 4.3 Errors of the LDG method (case 2) for example 1 for different $\alpha$ with $\varepsilon=0.01$ (left) and for different $\varepsilon$ with $\alpha=0.6$ (right).

\begin{tabular}{crc}
$\alpha$ & $e(f, 0.01)$ & $e_{r}(f, 0.01)$ \\
\hline 0.05 & $5.20 \times 10^{-5}$ & $5.18 \times 10^{-5}$ \\
0.1 & $1.26 \times 10^{-4}$ & $1.26 \times 10^{-4}$ \\
0.3 & $7.66 \times 10^{-4}$ & $7.66 \times 10^{-4}$ \\
0.7 & $6.55 \times 10^{-3}$ & $6.55 \times 10^{-3}$ \\
0.9 & $1.42 \times 10^{-2}$ & $1.42 \times 10^{-2}$ \\
0.95 & $1.67 \times 10^{-2}$ & $1.67 \times 10^{-2}$ \\
\hline
\end{tabular}

\begin{tabular}{crr}
\hline & & \\
$\varepsilon$ & $e(f, \varepsilon)$ & $e_{r}(f, \varepsilon)$ \\
\hline 0.0005 & 0.004143 & 0.004142571 \\
0.001 & 0.004142 & 0.004142483 \\
0.002 & 0.004142 & 0.004141573 \\
0.004 & 0.004141 & 0.004141178 \\
0.008 & 0.004146 & 0.004146441 \\
0.016 & 0.004153 & 0.004152914 \\
0.032 & 0.004166 & 0.004166003 \\
0.064 & 0.004209 & 0.004208976 \\
\hline
\end{tabular}




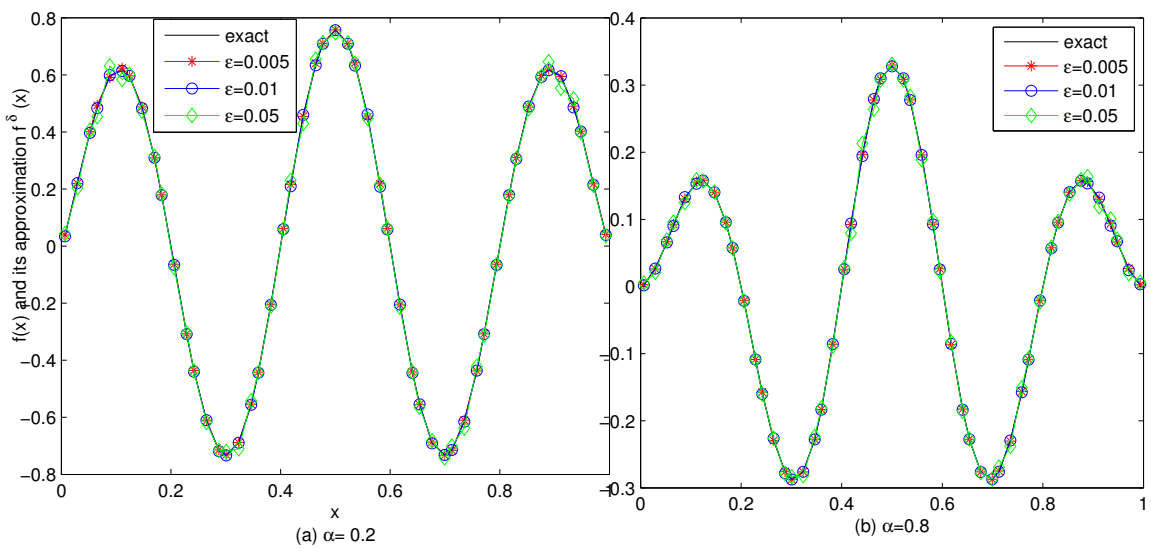

Fig. 4.2 The exact and approximate $f$ for Example 4.2

Table 4.4 Numerical results of Example 4.2 for different $\alpha$ with $\varepsilon=0.01$

\begin{tabular}{ccccc}
\hline$\alpha$ & $e(f, 0.01)$ of $[27]$ & $e(f, 0.01)$ & $e_{r}(f, 0.01)$ of $[27]$ & $e_{r}(f, 0.01)$ \\
\hline 0.05 & 0.0198 & 0.005997 & 0.0308 & 0.005381 \\
0.1 & 0.0180 & 0.003882 & 0.0306 & 0.003822 \\
0.3 & 0.0132 & 0.003702 & 0.0323 & 0.005222 \\
0.7 & 0.0079 & 0.002768 & 0.0377 & 0.007661 \\
0.9 & 0.0060 & 0.001415 & 0.0393 & 0.005400 \\
0.95 & 0.0055 & 0.001237 & 0.0396 & 0.005110 \\
\hline
\end{tabular}

Table 4.5 Numerical results of Example 4.2 for different $\varepsilon$ with $\alpha=0.6$

\begin{tabular}{ccccccc}
\hline & $e(f, \varepsilon)$ of [27] & $e(f, \varepsilon)$ & $e_{r}(f, \varepsilon)$ of $[27]$ & $e_{r}(f, \varepsilon)$ & Order of [27] & Order \\
\hline 0.0005 & 0.0024 & 0.000121 & 0.0096 & 0.000285 & - & - \\
0.001 & 0.0031 & 0.000249 & 0.0128 & 0.000585 & 0.4 & 1.0 \\
0.002 & 0.0043 & 0.000657 & 0.0176 & 0.001544 & 0.5 & 1.4 \\
0.004 & 0.0060 & 0.000974 & 0.0243 & 0.002288 & 0.5 & 0.6 \\
0.008 & 0.0082 & 0.001943 & 0.0332 & 0.004564 & 0.4 & 1.0 \\
0.016 & 0.0110 & 0.004485 & 0.0448 & 0.010533 & 0.4 & 1.2 \\
0.032 & 0.0146 & 0.010353 & 0.0606 & 0.024314 & 0.4 & 1.2 \\
0.064 & 0.0207 & 0.020734 & 0.0841 & 0.048695 & 0.5 & 1.0 \\
\hline
\end{tabular}

accessible, we first solve a direct problem using a suitable LDG method to obtain the input data $g$ and solve the inverse problem. The numerical results for various noise levels $\varepsilon=0.005,0.01,0.05$ for $\alpha=0.2,0.8$ are shown in Fig. 2. In Table 4.4, we compare the numerical errors for the method proposed in [27] with the proposed LDG method for different $\alpha$ with a fixed $\varepsilon=0.01$. In Table 4.5, we compare the numerical errors and convergence orders for the proposed method in [27] with the proposed LDG method for different $\varepsilon$ with a fixed $\alpha=0.6$. Without applying any regularization methods, our results are better than reported in [27]. 


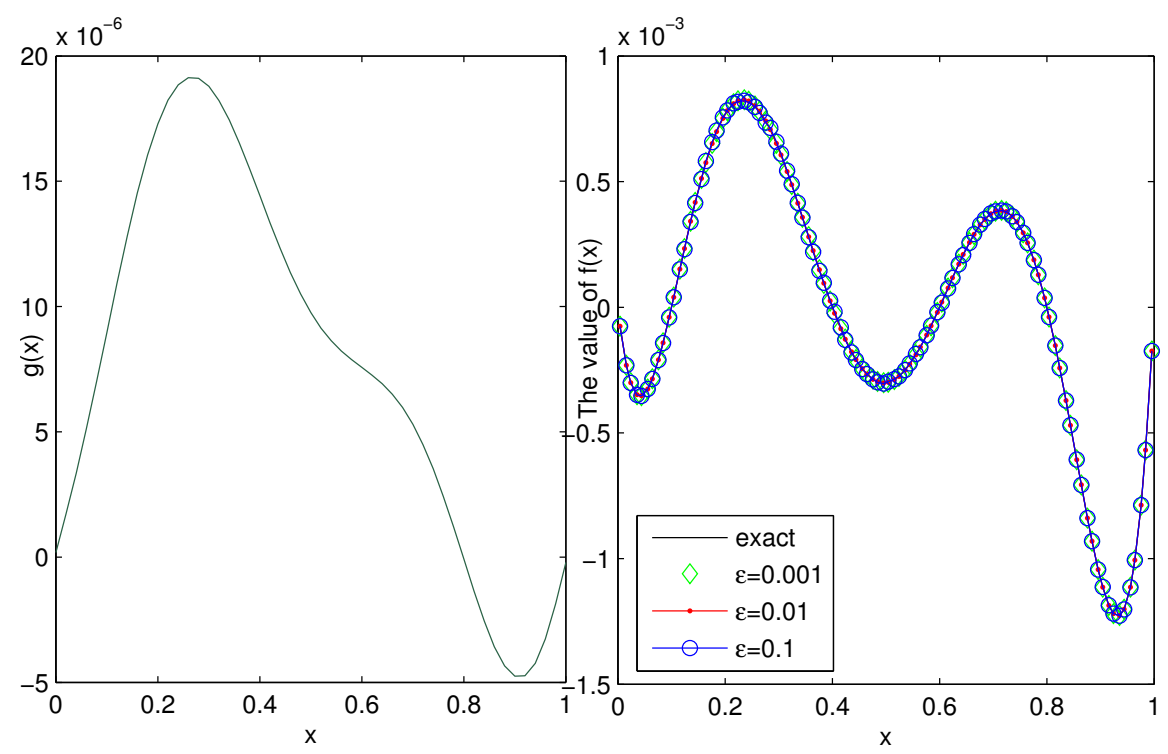

Fig. 4.3 Results of Example 4.3 with $\alpha=0.95$

Example 4.3 Take $f(x)=x(x-0.1)(x-0.4)(x-0.6)(x-0.8)(x-1)$. Since the exact solution of this problem is not accessible, we first solve a direct problem using a suitable LDG method to obtain the input data $g$ and solve the inverse problem. Numerical results for $\alpha=0.95$ with various noise levels $\varepsilon=0.1 \%, 1 \%, 10 \%$ are presented in Fig. 3 in which $e_{r}(f, \varepsilon)=0.000178099,0.000560097,0.004863713$. Without applying any regularization methods, our results are in good agreement with the results of [34].

Example 4.4 Consider a continuous piecewise smooth function, namely

$$
f(x)= \begin{cases}2 x, & x \in[0,0.5], \\ -2 x+2, & x \in(0.5,1] .\end{cases}
$$

Numerical results for $\alpha=0.95$ with various noise levels $\varepsilon=0.1 \%, 1 \%, 10 \%$ are presented in Fig. 4 in which $e_{r}(f, \varepsilon)=0.000175441,0.002526905,0.019711478$. Without applying any regularization methods, our results are in good agreement with the results of [34].

Example 4.5 This example involves reconstructing a discontinuous function given as

$$
f(x)= \begin{cases}0, & x \in[0,0.2] \\ 1, & x \in(0.2,0.4] \\ 0, & x \in(0.4,0.6] \\ -1, & x \in(0.6,0.8] \\ 0, & x \in(0.8,1] .\end{cases}
$$



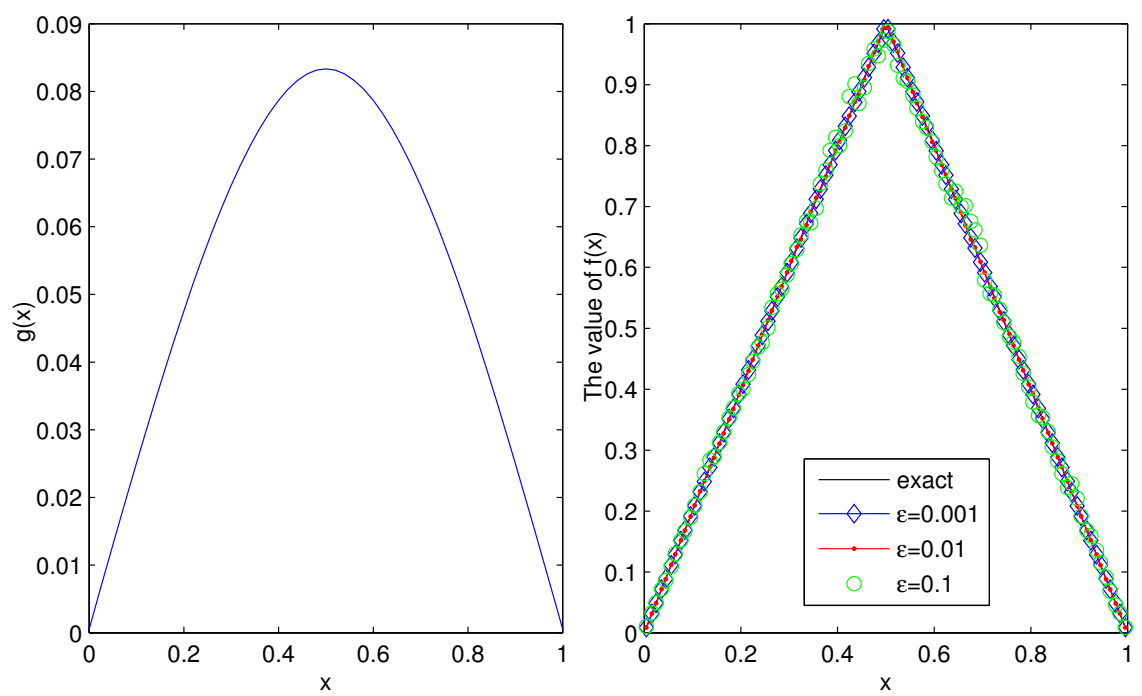

Fig. 4.4 Results of Example 4.4 with $\alpha=0.95$

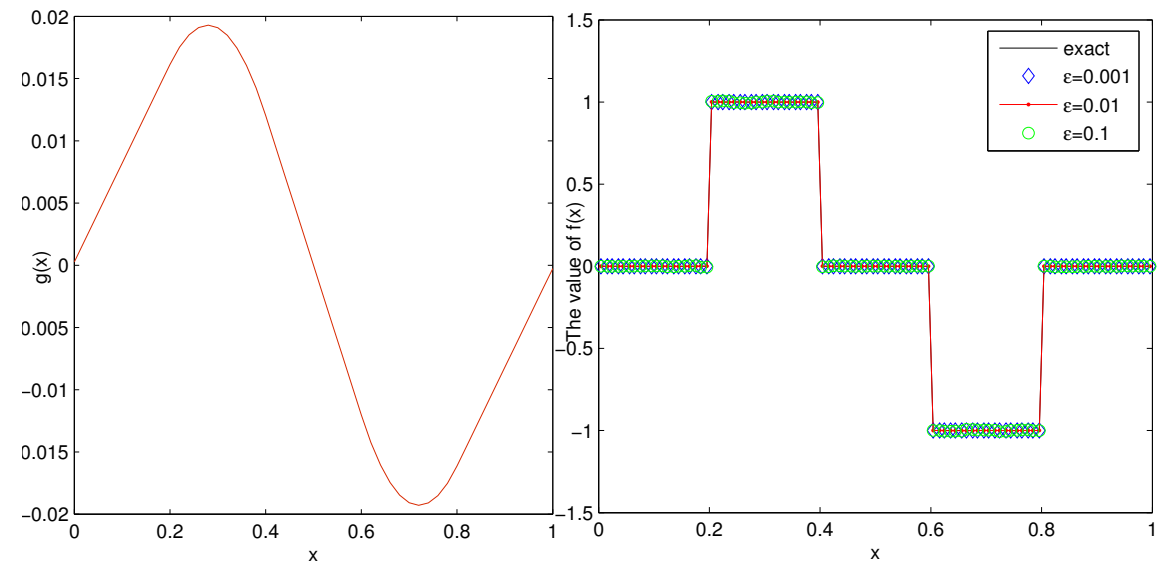

Fig. 4.5 Results of Example 4.5 with $\alpha=0.95$.

Numerical results for $\alpha=0.95$ with various noise levels $\varepsilon=0.1 \%, 1 \%, 10 \%$ are presented in Fig. 5 in which $e_{r}(f, \varepsilon)=0.000048381,0.000456007,0.004768183$. Without applying any regularization methods, our results are in good agreement with the results of [34]. 


\section{Appendix}

We seek to explain why our proposed method need not regularization method. Consider the time-fractional diffusion equation (1.1), with $a(x)=1$ and $c(x)=0$ (as we consider in examples 4.1 and 4.3-4.5). Let us decompose the domain of the problem into cells of equal length $h$ and choose for local basis functions of $\mathbb{P}^{1}\left(I_{j}\right)$ the monomial basis functions [20]. Solving (3.4), we have

$$
K_{11}=K_{22}=\operatorname{diag}\left(h, \frac{h}{3}, \ldots, h, \frac{h}{3}\right), \quad \bar{K}_{12}=\bar{K}_{21}=\operatorname{diag}(Z, \ldots, Z), \quad Z=\left[\begin{array}{ll}
0 & 0 \\
2 & 0
\end{array}\right],
$$

therefore $\bar{K}_{12} K_{22}^{-1} \bar{K}_{21} K_{22}^{-1}=(0)_{2 N \times 2 N}, K_{m}=I$ and

$$
A_{M} F=G-\beta G_{1}+\beta \bar{K}_{12} K_{22}^{-1} G_{2} .
$$

$A_{M}$ has a complex structure and we are not able to find a closed form for it. In Table 5.1 , we report $\left\|A_{M}^{-1}\right\|_{2}$ for different values of $\alpha$. Obviously $\left\|A_{M}^{-1}\right\|_{2}$ has a reasonable size and therefore in examples 4.1 and 4.3-4.5 numerical solutions are not sensitive with respect to the perturbation in the initial data.

Table 5.1 $\left\|A_{M}^{-1}\right\|_{2}$ for different values of $\alpha$

\begin{tabular}{cccccccccc}
\hline$\alpha$ & 0.1 & 0.2 & 0.3 & 0.4 & 0.5 & 0.6 & 0.7 & 0.8 & 0.9 \\
\hline$\left\|A_{M}^{-1}\right\|_{2}$ & 2.2842 & 4.6703 & 8.6683 & 15.039 & 24.861 & 39.783 & 62.628 & 98.208 & 154.09 \\
\hline
\end{tabular}

In table 5.2, we show $L^{2}$-norm of matrices $G, A_{M}^{-1} G$ and $A_{M}^{-1}$ for example 4.2. Obviously, $\left\|A_{M}^{-1} G^{\delta}\right\|_{2}$ is small and $\left\|A_{M}^{-1}\right\|_{2}$ has a reasonable size. Therefore numerical solutions are not sensitive with respect to the perturbation in the initial data. Therefore, we need not any regularization method.

Table 5.2 Norm 2 of some matrices for example 4.2 for different $\alpha$ with $\varepsilon=0.01$ (left) and for different $\varepsilon$ with $\alpha=0.6$ (right).

\begin{tabular}{|c|c|c|c|}
\hline 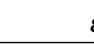 & $\|G\|_{2}$ & $\left\|A_{M}^{-1} G\right\|_{2}$ & $\left\|A_{M}^{-1}\right\|_{2}$ \\
\hline 0.0005 & $9.4606 \times 10^{-5}$ & 0.0099594 & 289.24 \\
\hline 0.001 & $9.4606 \times 10^{-5}$ & 0.0099593 & 289.24 \\
\hline 0.002 & $9.4608 \times 10^{-5}$ & 0.0099596 & 289.24 \\
\hline 0.004 & $9.4606 \times 10^{-5}$ & 0.0099593 & 289.24 \\
\hline 0.008 & $9.4604 \times 10^{-5}$ & 0.0099597 & 289.24 \\
\hline 0.016 & $9.4601 \times 10^{-5}$ & 0.0099590 & 289.24 \\
\hline 0.032 & $9.4612 \times 10^{-5}$ & 0.0099586 & 289.24 \\
\hline 0.064 & $9.4609 \times 10^{-5}$ & 0.0099596 & 289.24 \\
\hline
\end{tabular}

\begin{tabular}{cccc}
\hline & & & \\
$\alpha$ & $\|G\|_{2}$ & $\left\|A_{M}^{-1} G\right\|_{2}$ & $\left\|A_{M}^{-1}\right\|_{2}$ \\
\hline 0.05 & $1.9846 \times 10^{-4}$ & 0.40753 & 5.8379 \\
0.1 & $1.8721 \times 10^{-4}$ & 0.29199 & 11.371 \\
0.3 & $1.4698 \times 10^{-4}$ & 0.07701 & 64.902 \\
0.7 & $8.0296 \times 10^{-5}$ & 0.00501 & 436.65 \\
0.9 & $5.7313 \times 10^{-5}$ & 0.00129 & 965.3 \\
0.95 & $5.2690 \times 10^{-5}$ & 0.00092 & 1169.8 \\
\hline
\end{tabular}

The sequel of this appendix is devoted to investigating the discrete Picard condition [10] in order to show that our proposed method need not any regularization. Here we just investigate Example 4.2. Similar results obtain for Examples 4.3-4.5. We 

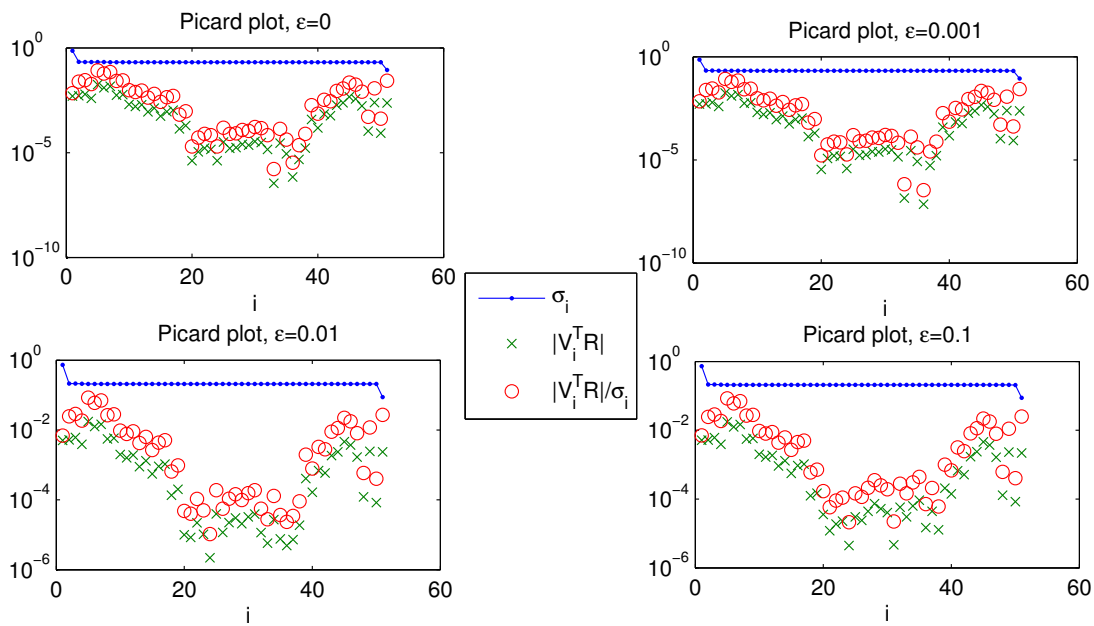

Fig. 5.1 Picard plot for Example 4.2 $(\alpha=0.1)$.
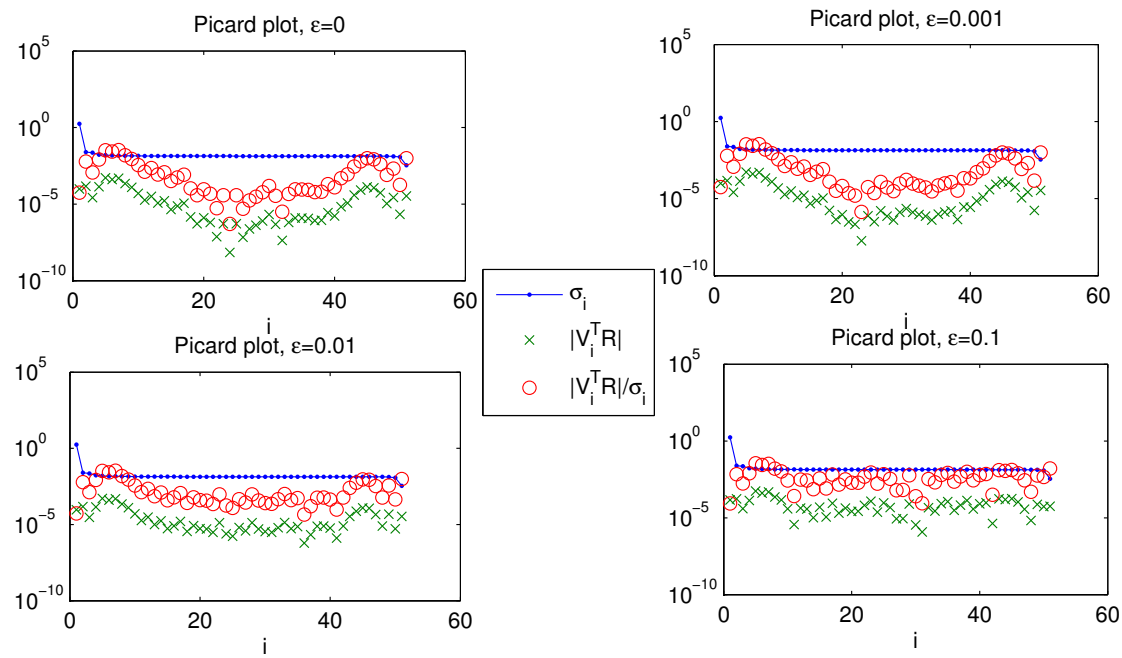

Fig. 5.2 Picard plot for Example $4.2(\alpha=0.6)$.

use the MATLAB codes developed by Hansen [11] to prepare Picard plots for both the unperturbed and the perturbed data with various noise levels $\varepsilon=0.1 \%, 1 \%, 10 \%$ which presented in Figures 5.1-5.3 for $\alpha=0.1,0.6,0.95$, respectively. In all cases, the Fourier coefficients $V_{i}^{T} R$ decay to zero faster than the $\sigma_{i}$. Here, $\left(\sigma_{i}, V_{i}\right)$ 's are the pair of singular values and corresponding (left) singular vectors of matrix $A_{M}$ and $R$ is the right-hand side of the linear system which will be solved, i.e. $A_{M} F=R:=$ $K_{M} G-\beta G_{1}+\beta \bar{K}_{12} K_{22}^{-1} G_{2}$. Therefore, according to the discrete Picard condition, our method need not any regularization. 


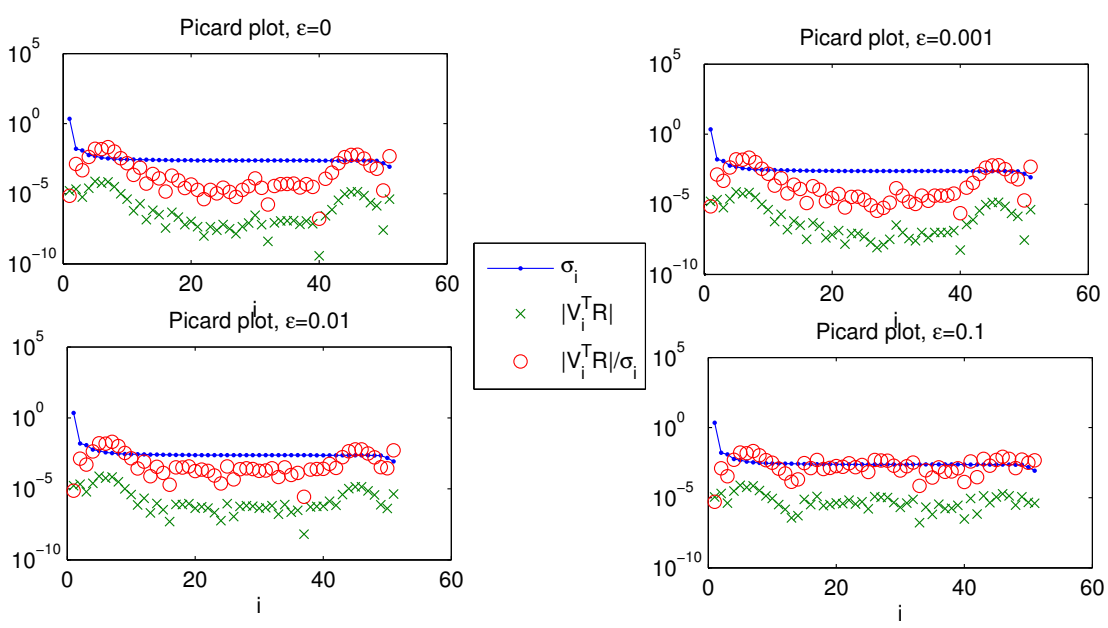

Fig. 5.3 Picard plot for Example $4.2(\alpha=0.95)$.

\section{Conclusion}

In this paper, we have developed a local discontinuous Galerkin finite element method for solving an inverse source problem for the time-fractional diffusion equation. Applying this method without using any regularization, we obtain stable and accurate numerical approximations to the space-dependent source term using an additional condition. The numerical stability and convergence of the method have been investigated and theoretically proven. Several examples with smooth or none-smooth data are given and the numerical results demonstrate the reliability and efficiency of the proposed method.

\section{References}

1. Boyd, S. and Vandenbergh, L.: Convex Optimization. Cambridge University Press, 2004.

2. Cheng, J., Nakagawa, J., Yamamoto, M., Yamazaki, T.: Uniqueness in an inverse problem for a onedimensional fractional diffusion equation. Inverse Probl. 25, 115002 (2009)

3. Cockburn, B., Shu, C.W.: The local discontinuous Galerkin method for time-dependent convectiondiffusion systems. SIAM J. Numer. Anal. 35, 2440-2463 (1998)

4. Cockburn, B., Shu, C.W.: The Runge-Kutta discontinuous Galerkin method for conservation laws V: multidimensional systems. J. Comput. Phys. 141, 199-224 (1998)

5. Cockburn, B., Kanschat, G., Perugia, I., Schotzau, D.: Superconvergence of the local discontinuous Galerkin method for elliptic problems on cartesian grids. SIAM J. Numer. Anal. 39, 264-285 (2001)

6. Deng, W.H.: Finite element method for the space and time fractional Fokker-Planck equation. SIAM J. Numer. Anal. 47, 204-226 (2008)

7. Deng, W.H., Hesthaven, J.S.: Local discontinuous Galerkin methods for fractional diffusion equations. Math. Modelling Numer. Anal. 47, 1845-1864 (2013)

8. Deng, W., Hesthaven, J.S.: Local discontinuous Galerkin methods for fractional ordinary differential equations, in BIT, 55(4), 967-985 (2015)

9. Geng, F.Z., Lin, Y.Z.: Application of the variational iteration method to inverse heat source problems. Comput. Math. Appl. 58(11-12), 2098-2102 (2009)

10. Hansen, P. C.: The discrete Picard condition for discrete ill-posed problems. BIT. 30, 658-672 (1990) 
11. Hansen. P. C.: Regularization Tools (A Matlab Package for Analysis and Solution of Discrete Ill-Posed Problems). Numer. Algor. 6, 1-35 (1994)

12. Jiang, Y.J., Ma, J.T.: High-order finite element methods for time-fractional partial differential equations. J. Comput. Appl. Math. 235(11), 3285-3290 (2011)

13. Li, C.Z., Chen, Y.: Numerical approximation of nonlinear fractional differential equations with subdiffusion and superdiffusion. Comput. Math. Appl. 62, 855-875 (2011)

14. Lin, Y.M., Xu, C.J.: Finite difference/spectral approximations for the time-fractional diffusion equation. J. Comput. Phys. 225, 1533-1552 (2007)

15. Liu, J.J., Yamamoto, M.: A backward problem for the time-fractional diffusion equation. Appl. Anal. 89, 1769-1788 (2010)

16. Murio, D.A.: Stable numerical solution of a fractional-diffusion inverse heat conduction problem Comput. Math. Appl. 53, 1492-1501 (2007)

17. Murio, D.A.: Time fractional IHCP with Caputo fractional derivatives. Comput. Math. Appl. 56, $2371-$ $2381(2008)$

18. Murio, D.A.: Stable numerical evaluation of Grünwald-Letnikov fractional derivatives applied to a fractional IHCP. Inverse Probl. Sci. Eng. 17, 229-243 (2009)

19. Qian, Z:: Optimal modified method for a fractional-diffusion inverse heat conduction problem. Inverse Probl. Sci. Eng. 18, 521-533 (2010)

20. Riviére, B.: Discontinuous Galerkin Methods for Solving Elliptic and Parabolic Equations: Theory and Implementation. Rice University, Houston, Texas (2008)

21. Rundell, W., Xu, X., Zuo, L.H.: The determination of an unknown boundary condition in a fractional diffusion equation. Appl. Anal. 92, 1-16 (2012)

22. Sakamoto, K., Yamamoto, M.: Initial value/boundary value problems for fractional diffusion-wave equations and applications to some inverse problems. J. Math. Anal. Appl. 382(1), 426-447 (2011)

23. Shidfar, A., Babaei, A., Molabahrami, A.: Solving the inverse problem of identifying an unknown source term in a parabolic equation. Comput. Math. Appl. 60(5), 1209-1213 (2010)

24. Tuan, V.K.: Inverse problem for fractional diffusion equation. Fractional Calculus Appl. Anal. 14 31-55 (2011)

25. Wei, H., Chen, W., Sun, H.G., Li, X.C.: A coupled method for inverse source problem of spatial fractional anomalous diffusion equations. Inverse Probl. Sci. Eng. 18, 945-956 (2010)

26. Wang, J.G., Zhou, Y.B., Wei, T.: Two regularization methods to identify a space-dependent source for the time-fractional diffusion equation. Appl. Numer. Math. 68, 39-57 (2013)

27. Wei, T., Wang, J.G.: A modified quasi-boundary value method for an inverse source problem of the time-fractional diffusion equation. Appl. Numer. Math. 78, 95-111 (2014)

28. Wei, L.L., Zhang, X.D., He, Y.N.: Analysis of a local discontinuous Galerkin method for timefractional advection-diffusion equations. Int. J. Numer. Meth. Heat \& Fluid Flow. 23, 634-648 (2013)

29. Wei, T., Zhang, Z.Q.: Reconstruction of a time-dependent source term in a time-fractional diffusion equation. Eng. Anal. Boundary Elem. 37, 23-31 (2013)

30. Wei, T., Zhang, Z.Q.: Stable numerical solution to a Cauchy problem for a time fractional diffusion equation. Eng. Anal. Boundary Elem. 40, 128-137 (2014)

31. Xu, Q., Hesthaven, J.S.: Discontinuous Galerkin method for fractional convection-diffusion equations. To appear in SIAM J. Numer. Anal.

32. Xu, Y., Shu, C.W.: Local Discontinuous Galerkin method for the Camassa-Holm equation. SIAM J Numer. Anal. 46, 1998-2021 (2008)

33. Yeganeh, S., Mokhtari, R., Fouladi, S.: A local discontinuous Galerkin method for solving an inverse source problem of the time-fractional diffusion equation. Submitted.

34. Zhang, Z.Q., Wei, T.: Identifying an unknown source in time-fractional diffusion equation by a truncation method. Appl. Math. Comput. 219, 5972-5983 (2013)

35. Zheng, G.H., Wei, T.: Spectral regularization method for a Cauchy problem of the time fractional advection-dispersion equation. J. Comput. Appl. Math. 233, 2631-2640 (2010)

36. Zheng, G.H., Wei, T.: A new regularization method for Cauchy problem of the fractional diffusion equation. Adv. Comput. Math. 36, 377-398 (2012) 